\title{
PERE: Una nueva herramienta para valorar el reconocimiento de las emociones básicas y su aplicación en la esquizofrenia
}

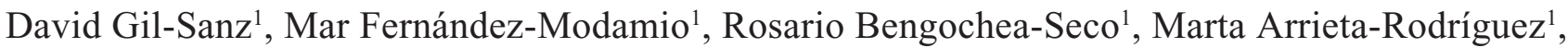 \\ Eduardo González-Fraile ${ }^{2}$, Gabriela Pérez-Fuentes ${ }^{3}$, Bárbara García-Polavieja ${ }^{1}$, Manuel Martín- \\ Carrasco $^{2}$, Juan Gómez de Tojeiro-Roce ${ }^{1}$, Borja Santos-Zorrozúa ${ }^{4}$ \\ ${ }^{1}$ Centro Hospitalario Padre Menni, Santander, España \\ ${ }^{2}$ Instituto de Investigaciones Psiquiátricas, Bilbao, España \\ ${ }^{3}$ University College London, London, UK \\ ${ }^{4}$ Universidad del País Vasco, Vizcaya, España
}

ERAT: A new tool to assess basic emotions recognition and its application in schizophrenia

\begin{abstract}
Research about emotion recognition in schizophrenia has shown that patients have a deficit in perception of negative emotions. There are different tests to assess emotion recognition, but they have several methodological limitations. The aim of the present article was to develop a new tool to assess the six basic emotions. A study was done with 450 control subjects and 60 outpatients with schizophrenia. Fifty-six images were selected to form the test, eight for each basic emotion and eight neutral ones. All photographs had an accuracy higher than $89 \%$ and a test-retest reliability between 0.80 and 1.32 for controls, and between 0.61 and 1.92 for outpatients. Overall, data obtained show that the Emotion Recognition Assessment Test (ERAT) may be a good tool to assess emotion recognition in schizophrenia. This test is available for free in www.proyectoscores.es/pere.php
\end{abstract}

Keywords: Basic emotions; schizophrenia; social cognition; assessment.

Resumen: Los estudios sobre reconocimiento de emociones en esquizofrenia han demostrado que los pacientes presentan un déficit en la percepción de emociones negativas. Hay varias pruebas disponibles para evaluar el reconocimiento de emociones, pero tienen inconvenientes de tipo metodológico. El objetivo del presente artículo fue desarrollar un nuevo instrumento para la valoración de las emociones básicas. El estudio se llevó a cabo con 450 sujetos control y 60 pacientes con esquizofrenia. Se seleccionaron 56 imágenes para formar la prueba, 8 por cada emoción básica y 8 neutras. Todas las fotografías obtuvieron una precisión de reconocimiento superior al $89 \%$ y una fiabilidad test-retest entre 0.80 y 1 para los sujetos control, y entre 0.61 y 1 para los pacientes. En conjunto, estos resultados indican que la Prueba de Evaluación del Reconocimiento de Emociones (PERE), disponible gratuitamente en www.proyectoscores.es/pere.php, puede ser una buena herramienta para evaluar el reconocimiento de emociones en esquizofrenia.

Palabras clave: Emociones básicas; esquizofrenia; cognición social; evaluación.

\section{Introducción}

La cognición social se ha definido como los procesos y funciones que permiten a una persona entender las si-

Recibido: 07/10/16; aceptado: 24/01/17

Correspondencia: David Gil-Sanz, Centro Hospitalario Padre Menni, Avda. de Cantabria 52, 39012 Santander, España. Correo-e: crpsant@, mennisant.com tuaciones sociales, actuar en dichas situaciones y beneficiarse de las interacciones con los demás (Corrigan y Penn, 2001; Pinkham et al., 2014). Se han propuesto cuatro dominios que integran la cognición social: el procesamiento emocional, la teoría de la mente, el estilo atribucional y la percepción social (Pinkham et al., 2014). El procesamiento emocional se refiere a la capacidad de percibir, entender y manejar de manera adecuada la información emocional, tanto en relación con uno 
mismo como con los demás (Green et al., 2008). La teoría de la mente se define como la capacidad de identificar los estados mentales de otras personas, como sus pensamientos, creencias e intenciones, y tenerlos en cuenta para explicar y predecir su conducta mientras que el estilo atribucional hace referencia al modo en que las personas explican los acontecimientos positivos y negativos que les suceden (Green et al., 2008). Por último, la percepción social implica la valoración y comprensión de las reglas y roles sociales que se dan en las situaciones sociales, y la capacidad de adecuar el propio comportamiento en función de estos elementos (Green et al., 2008).

En los últimos años se han publicado numerosos estudios sobre cognición social en esquizofrenia. En conjunto, estos estudios demuestran que las personas con esquizofrenia realizan un mal manejo de los dominios que integran la cognición social (Penn, Sanna, y Roberts, 2008), y que el déficit observado se relaciona de manera significativa con la competencia social, el desempeño psicosocial y la calidad de vida (Couture, Penn, y Roberts, 2006; Schmidt, Mueller, y Roder, 2011). Asimismo, estudios recientes vinculan la empatía con diferentes dominios de la cognición social, como la capacidad de manejar las emociones, la teoría de la mente y la percepción social (Green, Horan, y Lee, 2015). Dado que estos dominios están afectados en la esquizofrenia, los pacientes también muestran un déficit en su capacidad de ser empáticos con los demás (Alcalá, Camacho, Romero, y Blanco, 2013; Lee, Zaki, Harvey, Ochsner, y Green, 2011), lo que contribuye a que los pacientes con esquizofrenia no establezcan relaciones sociales adecuadas.

De los dominios anteriormente mencionados, los dos más estudiados en esquizofrenia han sido la teoría de la mente y el procesamiento emocional. Respecto a este último, diversos estudios han constatado que los pacientes con esquizofrenia tienen un déficit en la percepción de las emociones de tipo negativo (Savla, Vella, Armstrong, Penn, y Twamley, 2013; Weisberger et al., 2015). Este déficit se ha relacionado con diferentes aspectos del funcionamiento social, tales como la capacidad de autocuidado (Pan, Chen, Chen, y Liu, 2009), las habilidades para mantener una conversación (Hooker y Park, 2002), los malos entendidos en las relaciones interpersonales, $\mathrm{o}$ la presencia de comportamientos socialmente inadecuados (Pinkham y Penn, 2006). Se ha observado en pacientes que nunca han tomado medicación (Bediou et al., 2012), se mantiene estable a lo largo del curso de la enfermedad, y es independiente de la presencia de sintomatología, por lo que se ha propuesto como un marcador de riesgo de la esquizofrenia (Horan et al., 2012). Asi- mismo, en sujetos de alto riesgo, el déficit en el reconocimiento de algunas emociones negativas, como el miedo, podría predecir la transición a la psicosis (Allot et al., 2015).

Lo comentado anteriormente pone de relieve la importancia de contar con instrumentos adecuados que valoren la percepción de emociones en esquizofrenia. La mayoría de estudios han utilizado pruebas compuestas por fotografías de caras que expresan las diferentes emociones básicas (alegría, tristeza, enfado, miedo, sorpresa $\mathrm{y}$ asco). Entre estas pruebas cabe mencionar las siguientes: Ekman 60 Faces Test (EK-60F; Ekman y Friesen, 1976), Face Emotion Identification Task (FEIT; Kerr y Neale, 1993), Penn Emotion Recognition Test (ER-40; Gur et al., 2002), y Diagnostic Analysis of Non-verbal Accuracy 2 (DANVA 2; Nowicki y Carton, 1993).

Las dos primeras pruebas se elaboraron a partir de conjuntos de fotografías desarrollados por Izard (1971) y por Ekman y Friesen (Pictures of Facial Affect, 1976). Se trata de imágenes en blanco y negro en las que no se incluyen caras con expresión neutra. El EK-60F está compuesto por 60 fotografías de 10 actores (6 hombres y 4 mujeres) que se muestran a la persona durante 5 segundos cada una. Valora las 6 emociones básicas ofreciendo una puntuación global y una puntuación para cada emoción por separado. Hay disponible una versión informatizada. El FEIT lo forman 19 fotografías que se muestran a la persona durante 15 segundos cada una. Hay 15 fotografías que presentan emociones de tipo negativo y 4 que muestran emociones positivas (alegría y sorpresa). En esta prueba no hay ninguna fotografía relacionada con el asco pero sí se incluyen fotografías relacionadas con la vergüenza.

Por su parte, el ER-40 incluye 40 fotografías en color que expresan 4 emociones - alegría, tristeza, enfado y miedo-y expresiones neutras. Las imágenes están balanceadas por género, edad y raza, y para cada emoción hay 4 fotografías de alta intensidad y 4 de baja intensidad. Se trata de una prueba informatizada que proporciona puntuaciones precisas relacionadas con la identificación de cada emoción y con el tiempo medio de las respuestas. El ER-40 no incluye fotografías ni de sorpresa ni de asco. Por último, el DANVA 2 es una prueba compuesta por varios subtests que valoran diferentes elementos de la información social no verbal. El subtest denominado Adult Faces 2 incluye 24 fotografías ( 6 por cada emoción) de alegría, tristeza, enfado, y miedo de alta y baja intensidad, y el mismo número de imágenes de hombre y de mujer. Esta prueba no incluye fotografías de expresiones neutras.

En un artículo de 2002, Edwards y colaboradores (Edwards, Jackson, y Pattison, 2002) plantean que los 
instrumentos utilizados para valorar la percepción de emociones en esquizofrenia presentan limitaciones de tipo metodológico, tales como no incluir fotografías de las seis emociones básicas o fotografías de caras neutras. Para estos autores, la falta de caras neutras hace que no se pueda llegar a conclusiones claras sobre si existe un déficit en la percepción de emociones o si se trata de un déficit más general que afecta al reconocimiento de rasgos faciales (Bortolon, Capdevielle, y Raffard, 2015).

Asimismo, Edwards y colaboradores comentan que algunas de las fotografías utilizadas no presentan ningún dato de validez. En un estudio posterior, Palermo y Coltheart (2004) analizaron 294 fotografías seleccionadas de los conjuntos Pictures of Facial Affect, 3D Facial Emotional Stimuli de la Universidad de Pensilvania y NinStim Face Stimulus Set (Tottenham et al., 2009). Mostraron las fotografías a 24 sujetos control y les pidieron que valorasen qué emoción expresaba cada fotografía. Los resultados indicaron que el porcentaje de acuerdo sobre la emoción expresada era muy bajo en relación con algunas de las fotografías. En concreto, había fotografías de miedo, tristeza o asco donde el porcentaje de acuerdo estaba por debajo del 10\%. A partir de estos datos, los autores concluyeron que la alegría es la emoción que se reconoce con mayor precisión (Palermo y Coltheart, 2004). Sin embargo, una explicación alternativa podría ser que aquellas fotografías en las que se alcanzaba un porcentaje de acuerdo inferior al $10 \%$ no expresaban realmente la emoción que los investigadores pretendían que expresaran. En pocas palabras, una fotografía de miedo en la que menos del 10\% de los sujetos reconocen esa emoción, ¿es realmente una fotografía de miedo?

Recientemente se han desarrollado nuevas pruebas para valorar las emociones básicas, incluyendo como materiales fotografías, vídeos y archivos de audio, pero con el objetivo de evaluar las diferencias en el reconocimiento de las emociones entre sujetos de la población general sin ningún trastorno psiquiátrico (Palermo, O'Connor, David, Irons y McKone, 2013; Keutmann, 2014; Wilhelm, Hildebrandt, Manske, Schacht y Sommer, 2014). Por este motivo, los estímulos incluidos tienen en su mayoría una alta complejidad, por lo que podrían no resultar adecuados para evaluar el reconocimiento de emociones en personas con trastorno mental grave. De hecho, los autores no conocen ningún estudio en el que alguna de estas pruebas se haya utilizado con personas con esquizofrenia.

Por tanto, teniendo en cuenta las observaciones de Edwards y colaboradores (Edwards et al., 2002), así como los datos de Palermo y Coltheart (2004), se estimó conveniente desarrollar un nuevo instrumento para la va- loración de la percepción de emociones en pacientes con esquizofrenia, denominado Prueba de Evaluación del Reconocimiento de Emociones (PERE). El objetivo principal era obtener un conjunto de fotografías que expresasen de manera clara las 6 emociones básicas.

\section{Método}

\section{Participantes}

El estudio se desarrolló en tres fases. En la primera fase se realizaron las fotografías que formarían parte de la prueba, mientras que en la segunda y tercera fases se analizaron las propiedades psicométricas de las fotografías con población control y con pacientes con esquizofrenia, respectivamente. Para la elaboración de las fotografías, se reclutaron 19 voluntarios (10 varones y 9 mujeres) de raza caucásica, trabajadores del Centro Hospitalario Padre Menni (Santander, España), con edades entre 30 y 50 años, y sin formación en artes escénicas. Todos recibieron una sesión de entrenamiento sobre las unidades faciales que configuran cada emoción básica (García Fernández-Abascal y Chóliz Montañés, 2001). Los participantes no recibieron ningún tipo de compensación económica ni de ningún otro tipo por su participación.

La segunda fase se llevó a cabo con población control, que se reclutó difundiendo el estudio en redes sociales y mediante correo electrónico, teniendo como único criterio de inclusión que los participantes tuvieran entre 18 y 65 años. En esta fase del estudio se reclutaron 450 sujetos. Cuatro quedaron excluidos, dos debido a que tenían una edad superior a 65 años y otros dos porque ofrecieron un patrón de respuestas azaroso (en concreto, etiquetaron la mayoría de las fotografías como neutras). Por tanto, la muestra final estuvo compuesta por 446 sujetos, 310 mujeres $(69.51 \%)$. La media de edad fue de 39.75 años y 279 de los participantes tenían estudios universitarios $(62.56 \%)$. La tercera y última fase del estudio se realizó con una muestra de 60 sujetos con un diagnóstico de esquizofrenia, 39 mujeres (65\%). La edad media fue de 44.03 años y 27 de los participantes $(45 \%)$ tenían estudios secundarios, entendiendo por tales ESO, BUP o un grado equivalente de FP. El diagnóstico fue realizado por su psiquiatra de referencia del Sistema Cántabro de Salud. Todos acudían a un Centro de Rehabilitación Psicosocial perteneciente al Centro Hospitalario Padre Menni. Las características sociodemográficas y clínicas de ambas muestras se presentan en la Tabla 1. Ni los sujetos control ni los pacientes recibieron compensación económica ni de ningún otro tipo por participar en el estudio. 
Con la finalidad de valorar la fiabilidad test-retest del instrumento PERE, la prueba se administró en dos ocasiones con 15 días de diferencia entre ambas. En el caso de los sujetos control, todos los sujetos que habían participado en la primera administración de la prueba recibieron un código para poder asociar sus respuestas de la primera y la segunda administración. En la primera administración habían participado 446 sujetos, de los que 325 participaron en la administración retest. En el caso de la muestra con esquizofrenia, de los 60 sujetos de la primera administración, 51 pacientes accedieron a que se les pasara de nuevo la prueba.

Tabla 1. Características sociodemográficas

\begin{tabular}{lcc}
\hline & $\begin{array}{c}\text { Fase } 1(n=446)^{\mathrm{a}} \\
n(\%)\end{array}$ & $\begin{array}{c}\text { Fase } 2(n=60)^{\mathrm{b}} \\
n(\%)\end{array}$ \\
\hline $\begin{array}{l}\text { Sexo } \\
\text { Hombre }\end{array}$ & $136(30.49 \%)$ & $21(35 \%)$ \\
Mujer & $310(69.51 \%)$ & $39(65 \%)$ \\
Educación & & \\
$\quad$ Primaria & $25(5.6 \%)$ & $24(40 \%)$ \\
Secundaria & $142(31.84 \%)$ & $27(45 \%)$ \\
$\quad$ Universidad & $279(62.56 \%)$ & $9(15 \%)$ \\
Edad (Media $\pm D T)$ & $39.75 \pm 12.06$ & $44.03 \pm 9.94$ \\
Edad de inicio de la & - & $24.25 \pm 6.24$ \\
enfermedad & & \\
Años de evolución & - & $20.05 \pm 10.87$ \\
\hline
\end{tabular}

Nota. ${ }^{\text {a }}$ Controles; ${ }^{\mathrm{b}}$ Pacientes

\section{Procedimiento}

Aspectos éticos. Los 19 sujetos reclutados para la realización de las fotografías firmaron un documento de consentimiento informado y un documento de cesión de derechos de imagen, documentos en los que accedían a prestar su imagen de manera voluntaria y autorizaban que dicha imagen pudiese ser utilizada en estudios científicos así como publicada en páginas web. Ambos documentos contemplaban la normativa española vigente (art. 5 lo 15/1999). Por su parte, los sujetos que participaron en el estudio a través de la página web eran informados de que sus datos serían utilizados con fines de investigación. Los datos que se recogieron fueron sexo, edad y nivel educacional, y en ningún caso se registraron datos identificativos o la dirección IP (Internet Protocol) de los participantes. Por último, la muestra de pacientes con esquizofrenia recibió una hoja de información con la descripción y objetivos del estudio y también firmaron un documento de consentimiento informado. El estudio fue aprobado por el CEISH (Comité de Ética para las
Investigaciones relacionadas con Seres Humanos) de la Universidad del País Vasco (EHU/UPV).

Primera fase. Realización de las fotografías. De cada participante se obtuvieron 28 fotografías, 4 de cada emoción básica (alegría, tristeza, enfado, sorpresa, miedo y asco) y 4 con expresión neutra. En total se tomaron 532 fotografías. Todas las fotografías fueron realizadas por un fotógrafo profesional a una distancia de metro y medio sobre un fondo blanco, con una cámara fotográfica Nikon DSLR, D7100. Las fotografías tenían un tamaño de 13 x 18 centímetros y una resolución de 180 PPP (puntos por pulgada). El objetivo final era que el instrumento PERE estuviera compuesto por 56 fotografías, ya que se consideró que era un número adecuado para que la persona que administre la prueba obtenga información suficiente sobre la capacidad del evaluado de reconocer cada emoción y que, al mismo tiempo, resulte una prueba no demasiado larga ni costosa para el sujeto evaluado. Con esta finalidad, se realizó una primera selección de 16 fotografías de cada emoción básica y 16 con expresión neutra, 8 de hombre y 8 de mujer, de acuerdo con la valoración de la calidad de las expresiones de cada fotografía realizada por dos investigadores entrenados. De esta manera, se formó un set inicial de 112 fotografías.

Segunda fase. Selección de las fotografías para el instrumento PERE. El objetivo de la segunda fase fue seleccionar las 56 fotografías que finalmente formarían parte del instrumento PERE. Se diseñó una página web de libre acceso en la que los sujetos participantes podían ver las 112 imágenes del set inicial sin límite de tiempo. Para cada imagen debían elegir la etiqueta que creían que se correspondía con la emoción que representaba la fotografía mediante un sistema de opción múltiple forzada (alegría, tristeza, enfado, sorpresa, miedo, asco, neutra). Las fotografías se presentaron en un orden aleatorio, que siempre fue el mismo para todos los sujetos. La selección se hizo teniendo en cuenta la precisión y la fiabilidad test-retest de cada fotografía.

Tercera fase. Administración del instrumento PERE. La tercera fase del estudio se realizó con las 56 fotografías seleccionadas en la fase previa, y se analizaron nuevamente la precisión y la fiabilidad test-retest en la muestra de 60 pacientes.

\section{Análisis estadísticos}

Como se ha comentado previamente, en las fases dos y tres del estudio se valoró la precisión y la fiabilidad test-retest. La precisión se expresó en términos de porcentaje, y se definió como el número de veces que una fotografía se asociaba con la emoción que teóricamente 
representaba dividido entre el número total de veces que se veía esa fotografía (446 en el caso de los sujetos control y 60 en el de los pacientes con esquizofrenia). La fiabilidad test-retest se valoró mediante el coeficiente Phi. Dado que no se recogieron los datos identificativos de los sujetos control, el estudio se volvió a difundir por correo electrónico y en redes sociales para recordar la participación en el retest. Asimismo, se compararon las puntuaciones entre sujetos control y pacientes con esquizofrenia para cada una de las emociones valoradas por el instrumento PERE mediante el análisis de varianza de medidas repetidas (ANOVA) con un factor entre sujetos: participante (control y paciente) y un factor de medidas repetidas: emoción (alegría, tristeza, enfado, sorpresa, miedo, asco y neutra). Las diferencias entre cada una de las emociones se analizaron mediante el ajuste de Bonferroni para comparaciones múltiples. El rango de puntuaciones para todas las emociones estaba entre 0 y 8 . Los análisis estadísticos se realizaron con el programa estadístico R (versión 3.0, 2013).

\section{Resultados}

En la Tabla 2 se recogen los datos de precisión y fiabilidad test-retest para cada fotografía, tanto en el caso de los sujetos control como en el de los pacientes con esquizofrenia. En la tabla solo se han incluido las 56 fotografías seleccionadas para formar parte del instrumento PERE. Como se observa en la tabla, en el caso de los sujetos control todas las fotografías seleccionadas presentaron una precisión por encima del $89 \%$ en el reconocimiento, y unos datos adecuados de fiabilidad test-retest, estando todos los valores entre 0.80 y 1 . En el caso de los pacientes con esquizofrenia, las puntuaciones más bajas de precisión se obtuvieron para las fotografías de miedo. Sin embargo, en algunas fotografías de alegría los pacientes obtuvieron una precisión mejor que los sujetos control; de hecho, la precisión fue del $100 \%$ en las fotografías 46, 53 y 55 . En cuanto a la fiabilidad test-retest, los valores del coeficiente Phi se situaron entre 0.61 y 1 .

Tabla 2. Precisión y fiabilidad test-retest para cada fotografía en sujetos control y pacientes

\begin{tabular}{|c|c|c|c|c|c|c|}
\hline \multirow[b]{2}{*}{ Foto $^{\mathrm{a}}$} & \multirow[b]{2}{*}{ Emoción } & \multirow[b]{2}{*}{$\mathrm{Sexo}^{\mathrm{b}}$} & \multicolumn{2}{|c|}{ Precisión ${ }^{1}$} & \multicolumn{2}{|c|}{ Phi } \\
\hline & & & $n=446^{\mathrm{c}}$ & $n=60^{\mathrm{d}}$ & $n=325^{\mathrm{c}}$ & $n=51^{\mathrm{d}}$ \\
\hline 1 & Tristeza & $\mathrm{H}$ & 93.2 & 91.7 & $0.92 * *$ & $0.92 * *$ \\
\hline 2 & Sorpresa & $\mathrm{H}$ & 97.4 & 100 & $0.97 * *$ & $0.97 * *$ \\
\hline 3 & Neutra & M & 97.3 & 81.7 & $0.98 * *$ & $0.98 * *$ \\
\hline 4 & Enfado & $\mathrm{H}$ & 94.9 & 80.0 & $0.93 * *$ & $0.93 * *$ \\
\hline 5 & Asco & $\mathrm{H}$ & 97.7 & 93.3 & $0.91 * *$ & $0.91 * *$ \\
\hline 6 & Sorpresa & $\mathrm{H}$ & 98.1 & 90.0 & $0.89 * *$ & $0.89 * *$ \\
\hline 7 & Miedo & M & 90.1 & 75.0 & $0.84 * *$ & $0.84 * *$ \\
\hline 8 & Asco & $\mathrm{H}$ & 98.5 & 81.7 & $1.00 * *$ & $1.00 * *$ \\
\hline 9 & Tristeza & $\mathrm{H}$ & 91.5 & 80.0 & $1.00 * *$ & $1.00^{* *}$ \\
\hline 10 & Sorpresa & M & 95.8 & 86.7 & $0.88 * *$ & $0.88 * *$ \\
\hline 11 & Alegría & $\mathrm{H}$ & 97.8 & 96.7 & $1.00 * *$ & $1.00 * *$ \\
\hline 12 & Alegría & M & 97.9 & 93.3 & $0.88 * *$ & $0.88^{* *}$ \\
\hline 13 & Enfado & M & 96.9 & 90.0 & $0.98 * *$ & $0.98^{* *}$ \\
\hline 14 & Asco & $\mathrm{H}$ & 95.5 & 91.7 & $0.83 * *$ & $0.83^{* *}$ \\
\hline 15 & Neutra & $\mathrm{H}$ & 95.7 & 93.3 & $1.00 * *$ & $1.00 * *$ \\
\hline 16 & Miedo & $\mathrm{H}$ & 90.0 & 35.0 & $0.84 * *$ & $0.84 * *$ \\
\hline 17 & Enfado & M & 96.4 & 90.0 & $0.96 * *$ & $0.96^{* *}$ \\
\hline 18 & Miedo & M & 89.8 & 55.0 & $0.80 * *$ & $0.80 * *$ \\
\hline 19 & Tristeza & M & 90.1 & 81.7 & $0.96 * *$ & $0.96^{* *}$ \\
\hline 20 & Alegría & M & 98.2 & 96.7 & $1.00 * *$ & $1.00 * *$ \\
\hline 21 & Asco & $\mathrm{H}$ & 95.5 & 76.7 & $0.97 * *$ & $0.97 * *$ \\
\hline 22 & Asco & M & 97.7 & 83.3 & $0.82 * *$ & $0.82 * *$ \\
\hline 23 & Miedo & $\mathrm{H}$ & 92.2 & 60.0 & $0.96 * *$ & $0.96^{* *}$ \\
\hline 24 & Alegría & $\mathrm{H}$ & 98.9 & 93.3 & $0.91 * *$ & $0.91 * *$ \\
\hline 25 & Miedo & M & 89.8 & 50.0 & $0.82 * *$ & $0.82 * *$ \\
\hline 26 & Tristeza & M & 91.9 & 75.0 & $0.98 * *$ & $0.98 * *$ \\
\hline 27 & Enfado & M & 98.2 & 96.7 & $0.98 * *$ & $0.98 * *$ \\
\hline 28 & Asco & M & 97.7 & 85.0 & $0.80 * *$ & $0.80 * *$ \\
\hline 29 & Alegría & M & 97.9 & 100 & $1.00 * *$ & $1.00^{* *}$ \\
\hline 30 & Tristeza & $\mathrm{H}$ & 91.0 & 35.0 & $0.97 * *$ & $0.97 * *$ \\
\hline
\end{tabular}


90 D. Gil, M. Fernández, R. Bengochea, M. Arrieta, E. González, G. Pérez, B. García, M. Martín, J. Gómez de Tojeiro, B. Santos

Tabla 2. Precisión y fiabilidad test-retest para cada fotografía en sujetos control y pacientes (Continuación)

\begin{tabular}{|c|c|c|c|c|c|c|}
\hline \multirow[b]{2}{*}{ Foto $^{a}$} & \multirow[b]{2}{*}{ Emoción } & \multirow[b]{2}{*}{$\mathrm{Sexo}^{\mathrm{b}}$} & \multicolumn{2}{|c|}{ Precisión $^{1}$} & \multicolumn{2}{|c|}{ Phi } \\
\hline & & & $n=446^{\mathrm{c}}$ & $n=60^{\mathrm{d}}$ & $n=325^{\mathrm{c}}$ & $n=51^{\mathrm{d}}$ \\
\hline 31 & Sorpresa & M & 94.0 & 95.0 & $0.84 * *$ & $0.84 * *$ \\
\hline 32 & Neutra & $\mathrm{H}$ & 94.9 & 93.3 & $0.98 * *$ & $0.98 * *$ \\
\hline 33 & Enfado & $\mathrm{H}$ & 93.7 & 98.3 & $0.89 * *$ & $0.89^{* *}$ \\
\hline 34 & Sorpresa & $\mathrm{H}$ & 96.7 & 88.3 & $0.90 * *$ & $0.90 * *$ \\
\hline 35 & Tristeza & $\mathrm{H}$ & 92.8 & 93.3 & $0.93 * *$ & $0.93 * *$ \\
\hline 36 & Neutra & $\mathrm{H}$ & 98.5 & 90.0 & $0.99 * *$ & $0.99 * *$ \\
\hline 37 & Miedo & $\mathrm{H}$ & 92.0 & 38.3 & $0.90 * *$ & $0.90 * *$ \\
\hline 38 & Tristeza & M & 96.9 & 95.0 & $0.88 * *$ & $0.88 * *$ \\
\hline 39 & Sorpresa & M & 97.1 & 90.0 & $0.94 * *$ & $0.94 * *$ \\
\hline 40 & Neutra & M & 95.4 & 90.0 & $0.99 * *$ & $0.99 * *$ \\
\hline 41 & Neutra & M & 93.1 & 86.7 & $0.98 * *$ & $0.98 * *$ \\
\hline 42 & Miedo & M & 90.2 & 61.7 & $0.87 * *$ & $0.87 * *$ \\
\hline 43 & Neutra & M & 96.6 & 93.3 & $1.00 * *$ & $1.00 * *$ \\
\hline 44 & Miedo & $\mathrm{H}$ & 90.4 & 76.7 & $0.94 * *$ & $0.94 * *$ \\
\hline 45 & Neutra & $\mathrm{H}$ & 95.3 & 96.7 & $1.00 * *$ & $1.00 * *$ \\
\hline 46 & Alegría & M & 97.8 & 100.0 & $1.00 * *$ & $1.00 * *$ \\
\hline 47 & Enfado & $\mathrm{H}$ & 94.2 & 91.7 & $0.93 * *$ & $0.93 * *$ \\
\hline 48 & Sorpresa & M & 98.7 & 95.0 & $0.97 * *$ & $0.97 * *$ \\
\hline 49 & Sorpresa & $\mathrm{H}$ & 98.5 & 80.0 & $0.84 * *$ & $0.84 * *$ \\
\hline 50 & Enfado & $\mathrm{H}$ & 96.8 & 95.0 & $0.90 * *$ & $0.90 * *$ \\
\hline 51 & Asco & M & 98.0 & 90.0 & $0.94 * *$ & $0.94 * *$ \\
\hline 52 & Asco & M & 98.0 & 83.3 & $1.00 * *$ & $1.00 * *$ \\
\hline 53 & Alegría & $\mathrm{H}$ & 97.5 & 100.0 & $1.00 * *$ & $1.00 * *$ \\
\hline 54 & Enfado & M & 95.3 & 90.0 & $0.87 * *$ & $0.87 * *$ \\
\hline 55 & Alegría & $\mathrm{H}$ & 97.2 & 100.0 & $0.93 * *$ & $0.93 * *$ \\
\hline 56 & Tristeza & M & 96.7 & 78.3 & $0.97 * *$ & $0.97 * *$ \\
\hline
\end{tabular}

Nota. ${ }^{1}$ Expresada en términos de porcentaje; ${ }^{a}$ Las fotografías se han puesto en el orden en que aparecen en el instrumento PERE;

${ }^{\mathrm{b}} \mathrm{H}$ : hombre, M: mujer; ${ }^{\mathrm{c}}$ Grupo control; ${ }^{\mathrm{d}}$ Pacientes. ${ }^{*} p<0.005 * * p<0.001$

El ANOVA mostró que se daba una interacción significativa entre el factor grupo y el factor emoción $[F(6,229)=19.9, p<0.001]$. Mediante el ajuste de Bonferroni para comparaciones múltiples se observó que había diferencias significativas entre ambas muestras en el reconocimiento de todas las emociones, salvo en la alegría (ver Tabla 3). Estas diferencias fueron especial-

Tabla 3. Puntuación media en el reconocimiento de cada emoción para los sujetos control y los pacientes, y ajuste de Bonferroni para comparaciones múltiples

\begin{tabular}{|c|c|c|c|c|}
\hline & Grupo control & Pacientes & & \\
\hline Emoción & Media $\pm D T$ & Media $\pm D T$ & gl & $F$ \\
\hline Alegría & $7.88 \pm 0.03$ & $7.78 \pm 0.06$ & 1 & 0.40 \\
\hline Tristeza & $7.56 \pm 0.06$ & $6.30 \pm 0.11$ & 1 & $54.20 * *$ \\
\hline Enfado & $7.82 \pm 0.05$ & $7.33 \pm 0.08$ & 1 & $9.25^{*}$ \\
\hline Sorpresa & $7.75 \pm 0.06$ & $7.22 \pm 0.10$ & 1 & $10.33^{*}$ \\
\hline Miedo & $7.37 \pm 0.11$ & $4.50 \pm 0.19$ & 1 & $107.14^{* *}$ \\
\hline Asco & $7.85 \pm 0.07$ & $6.85 \pm 0.12$ & 1 & $31.03 * *$ \\
\hline Neutra & $7.72 \pm 0.06$ & $7.25 \pm 0.11$ & 1 & $6.24^{*}$ \\
\hline
\end{tabular}

Nota. DT: desviación típica, gl: grados de libertad.

$* * p<0.01 ; * * * p<0.001$ mente significativas para las emociones de tristeza, miedo y asco. También se obtuvieron diferencias significativas para la identificación de las expresiones neutras. Como se observa en la Figura 1, la mayor diferencia entre grupos se dio en el reconocimiento del miedo.

Por otro lado, dado que había diferencias relevantes en el nivel educacional entre los sujetos control y los pacientes con esquizofrenia, se incluyó esta variable como covariable en el ANOVA de medidas repetidas. No se obtuvo una interacción significativa entre el factor emoción y el nivel educacional $[F(6,229)=0.8 \mathrm{~ns}]$.

\section{Discusión}

En el presente estudio se ha descrito el proceso de desarrollo de un nuevo instrumento para la valoración del reconocimiento de emociones en pacientes con esquizofrenia, denominado Prueba de Evaluación del Reconocimiento de Emociones (PERE). Un objetivo fundamental era que todas las fotografías que formasen parte de la prueba debían expresar de manera clara y precisa las seis emociones básicas. Los resultados obte- 


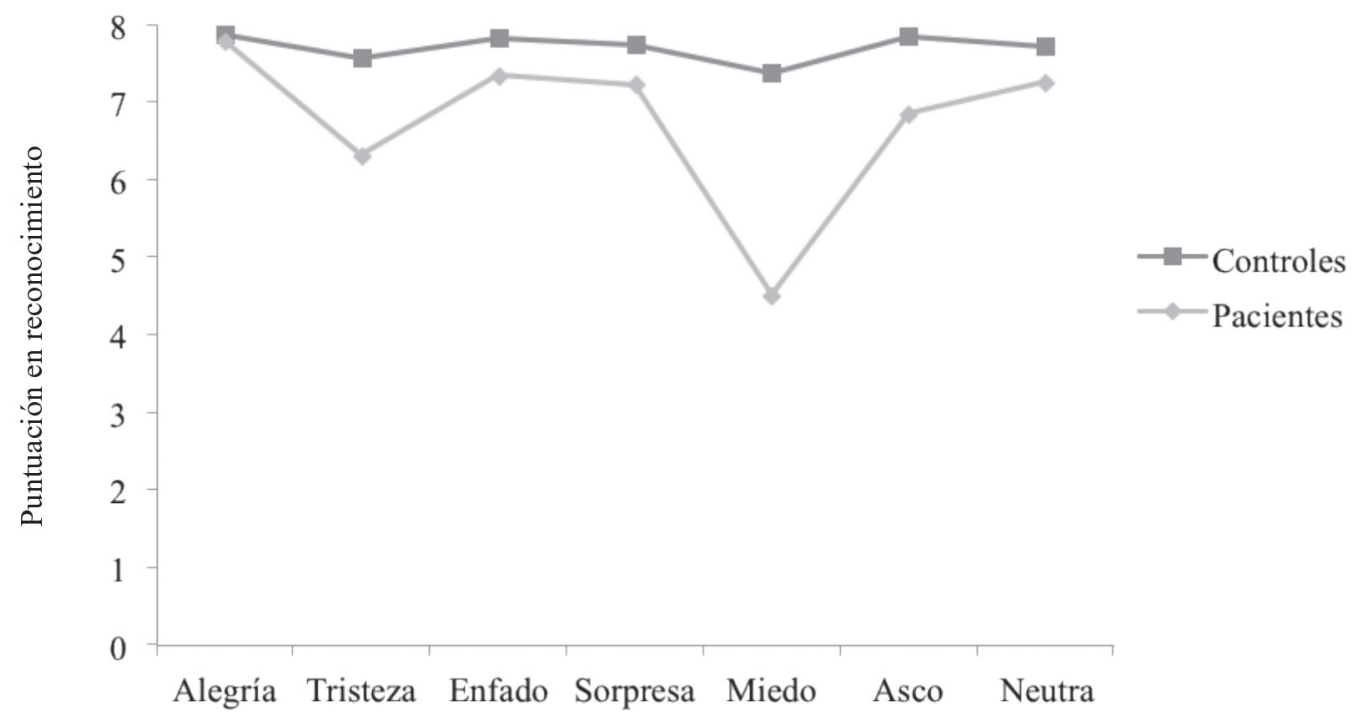

Figura 1. Comparación entre participantes controles y pacientes esquizofrénicos en relación con el reconocimiento de cada emoción básica

nidos en la muestra de sujetos control respecto a la precisión (por encima del 89\% para todas las fotografías) y la fiabilidad test-retest (entre.80 y 1) parecen indicar que las 56 fotografías seleccionadas cumplen esta condición. En los pacientes con esquizofrenia la precisión del reconocimiento de las emociones tuvo una mayor variabilidad, yendo desde el $38.3 \%$ para una de las fotografías de miedo hasta el 100\% para algunas imágenes de alegría. Los valores de la fiabilidad test-retest se situaron entre $.61 \mathrm{y} 1$.

El instrumento PERE es una prueba formada por 56 fotografías, 8 por cada emoción básica y 8 con expresión neutra. La prueba ofrece una puntuación individual para cada emoción de 0 a 8 , y una puntuación total de 0 a 56 . El tiempo de aplicación en pacientes con esquizofrenia es de aproximadamente 15 minutos. Como se mencionaba en la introducción, la inclusión de caras neutras puede contribuir a valorar si el déficit en el reconocimiento de emociones es un déficit específico o de carácter más general para el procesamiento de rasgos faciales. En el desarrollo del instrumento PERE también se ha tenido en cuenta que haya el mismo número de fotografías de hombre y de mujer, ya que esta característica puede facilitar el estudio de la influencia del sexo en la capacidad de reconocer una emoción. En sujetos control se ha observado que las mujeres tienen mayor habilidad para reconocer emociones y que éstas se identifican mejor en caras femeninas (Lewin y Herlitz, 2002; Palermo y Coltheart, 2004). En sujetos con esquizofrenia también se ha observado que las mujeres reconocen las emociones mejor que los hombres, especialmente aquellas de tipo negativo (Erol, Putgul, Kosger y Ersoy, 2013; Scholten, Aleman, Montagne y Kahn, 2005), si bien es un aspecto que requiere de mayor investigación.

Los resultados del instrumento PERE con los sujetos control indican que las emociones que mejor se reconocen son las de alegría, asco y enfado, mientras que el miedo parece ser la que peor se identifica. En todo caso, en todas las emociones se obtienen puntuaciones medias por encima de 7. Por su parte, los pacientes con esquizofrenia identifican mejor las emociones de alegría, enfado y sorpresa, y también es el miedo la emoción que peor se percibe (ver Tabla 2). En la comparación entre ambos grupos, se observan diferencias significativas en el reconocimiento de todas las emociones, salvo en la identificación de la alegría. Estos resultados están en línea con los observados en estudios previos, que mantienen que el reconocimiento de la alegría no está deteriorado en la esquizofrenia (Weisberger et al., 2015) y que el déficit en el reconocimiento de emociones está relacionado únicamente con las emociones de tipo negativo. Dentro de las emociones negativas, numerosos estudios también han encontrado que la emoción que peor perciben los pacientes con esquizofrenia es el miedo (Savla et al., 2013; Weisberger et al., 2015).

Como prueba de evaluación, la principal limitación del instrumento PERE es que solo incluye fotografías de personas de raza caucásica y con una edad comprendida entre los 30 y los 50 años. Asimismo, la muestra de pacientes con esquizofrenia estuvo integrada por 60 pacientes ambulatorios, por lo que los datos obtenidos podrían no ser generalizables a pacientes con otras características. 
En futuras investigaciones sería interesante utilizar el instrumento PERE en estudios que analicen la influencia del sexo en el reconocimiento de emociones, así como ver qué emociones se confunden entre sí, si ese fuera el caso. Por otro lado, futuros estudios podrían centrarse en aumentar los datos sobre las propiedades psicométricas del instrumento PERE aplicando el instrumento a sujetos control sanos, con un mayor control tanto del contexto de aplicación (p.ej., aplicación presencial) como del tiempo de exposición de las fotografías. De hecho, contar con un tiempo determinado para visualizar las 56 imágenes contribuiría a una mayor estandarización de la prueba. Por último, si bien el presente estudio se ha centrado en analizar la aplicación del instrumento PERE en pacientes con esquizofrenia, podría resultar de interés valorar su utilidad en pacientes con otras patologías que también estén asociadas a dificultades en la identificación de emociones, como el trastorno bipolar o el daño cerebral adquirido.

El instrumento PERE está disponible de manera gratuita en http://www.proyectoscores.es/pere.php

\section{Conflictos de intereses}

Los autores declaran que no existen conflictos de intereses

\section{Referencias}

Alcalá, V., Camacho, S., Romero, S. L., y Blanco, N. (2013). La falta de empatía: ¿Un síntoma específico de la esquizofrenia? Revista de Psicopatología y Psicología Clínica, 18, 73-80.

Allott, K. A., Rice, S., Bartholomeusz, C. F., Klier, C., Schlögelhofer, M., Schäfer, M. R., \& Amminger, G. P. (2015). Emotion recognition in unaffected first-degree relatives of individuals with first-episode schizophrenia. Schizophrenia Research, 161, 322-328.

Bediou, B., Brunelin, J., d'Amato, T., Fecteau, S., Saoud, M., Hénaff, M.-A., \& Krolak-Salmon, P. (2012). A comparison of facial emotion processing in neurological and psychiatric conditions. Frontiers in Psychology, 3, 98.

Bortolon, C., Capdevielle, D., \& Raffard, S. (2015). Face recognition in schizophrenia disorder: A comprehensive review of behavioral, neuroimaging and neurophysiological studies. Neuroscience and Biobehavioral Reviews, 53, 79-107.

Corrigan, P. W., \& Penn, D. L. (2001). Social cognition and schizophrenia. Portland, OR: APA.

Couture, S. M., Penn, D. L., \& Roberts, D. L. (2006). The functional significance of social cognition in schizophrenia: an overview. Schizophrenia Bulletin, 32, S44-63.

Edwards, J., Jackson, H. J., \& Pattison, P. E. (2002). Emotion recognition via facial expression and affective prosody in schizophrenia: a methodological review. Clinical Psychology Review, 22, 789-832.
Ekman, P., \& Friesen, W. V. (1976). Pictures of Facial Affect. Palo Alto, CA: Consulting Psychologists Press.

Erol, A., Putgul, G., Kosger, F., \& Ersoy, B. (2013). Facial emotion recognition in schizophrenia: the impact of gender. Psychiatry Investigation, 10, 69-74.

García Fernández-Abascal, E., y Chóliz-Montañés, M. (2001). Expresión facial de la emoción. Madrid: UNED.

Green, M. F., Horan, W. P., \& Lee, J. Social cognition in schizophrenia. Nature reviews. Neuroscience, 16, 620-631.

Green, M. F., Penn, D. L., Bentall, R., Carpenter, W. T., Gaebel, W., Gur, R. C., ... Hienssen, R. (2008). Social cognition in schizophrenia: an NIMH workshop on definitions, assessment, and research oportunities. Schizophrenia Bulletin, 34, 1211-1220.

Gur, R. E., McGrath, C., Chan, R. M., Schroeder, L., Turner, T., Turetsky, B. I., ... Gur, R. C. (2002). An fMRI study of facial emotion processing in patients with schizophrenia. The American Journal of Psychiatry, 159, 1992-1999.

Hooker, C., \& Park, S. (2002). Emotion processing and its relationship to social functioning in schizophrenia patients. $P s y$ chiatry Research, 112, 41-50.

Horan, W. P., Green, M. F., DeGroot, M., Fiske, A., Hellemann, G., Kee, K., ... Nuechterlein, K. H. (2012). Social cognition in schizophrenia, Part 2: 12-month stability and prediction of functional outcome in first-episode patients. Schizophrenia Bulletin, 38, 865-872.

Izard, C. (1971). The Face of Emotion. New York: Appleton-Century- Crofts.

Kerr, S. L., \& Neale, J. M. (1993). Emotion perception in schizophrenia: specific deficit or further evidence of generalized poor performance? Journal of Abnormal Psychology, 102, 312-318.

Keutmann, M. K., Moore, S. L., Savitt, A., \& Gur, R. C. (2015). Generating an item pool for translational social cognition research: methodology and initial validation. Behavior Research Methods, 47, 228-234.

Lee, J., Zaki, J., Harvey, P. O., Ochsner, K., \& Green, M. F. (2011). Schizophrenia patients are impaired in empathic accuracy. Psychological Medicine, 41, 2297-2304.

Lewin, C., \& Herlitz, A. (2002). Sex differences in face recognition-women's faces make the difference. Brain Cognition, 50, 121-128.

Nowicki, S., \& Carton, J. (1993). The measurement of emotional intensity from facial expressions. The Journal of Social Psychology, 13, 749-750.

Palermo, R., \& Coltheart, M. (2004). Photographs of facial expression: accuracy, response times, and ratings of intensity. Behavior Research Methods, Instruments, \& Computers : A Journal of the Psychonomic Society, Inc, 36, 634-638.

Palermo, R., O’Connor, K. B., Davis, J. M., Irons, J., \& McKone, E. (2013). New tests to measure individual differences in matching and labelling facial expressions of emotion, and their association with ability to recognise vocal emotions and facial identity. PloS One, 8, e68126.

Pan, Y.-J., Chen, S.-H., Chen, W. J., \& Liu, S.-K. (2009). Affect recognition as an independent social function determinant in schizophrenia. Comprehensive Psychiatry, 50, 443-452.

Penn, D. L., Sanna L. J., \& Roberts, D. L. (2008). Social cognition in schizophrenia: an overview. Schizophrenia Bulletin, 34, 408-411.

Pinkham, A. E., \& Penn, D. L. (2006). Neurocognitive and social 
cognitive predictors of interpersonal skill in schizophrenia. Psychiatry Research, 143, 167-178.

Pinkham, A. E., Penn, D. L., Green, M. F., Buck, B., Healey, K., \& Harvey, P. D. (2014). The social cognition psychometric evaluation study: results of the expert survey and RAND panel. Schizophrenia Bulletin, 40: 813-823.

Savla, G. N., Vella, L., Armstrong, C. C., Penn, D. L., \& Twamley, E. W. (2013). Deficits in domains of social cognition in schizophrenia: a meta-analysis of the empirical evidence. Schizophrenia Bulletin, 39, 979-992.

Schmidt, S. J., Mueller, D. R., \& Roder, V. (2011). Social cognition as a mediator variable between neurocognition and functional outcome in schizophrenia: empirical review and new results by structural equation modeling. Schizophrenia Bulletin, 37, S41-54.
Scholten, M. R. M., Aleman, A., Montagne, B., \& Kahn, R. S. (2005). Schizophrenia and processing of facial emotions: sex matters. Schizophrenia Research, 78, 61-67.

Tottenham, N., Tanaka, J. W., Leon, A. C., McCarry, T., Nurse, M., Hare, T. A., ... Nelson, C. (2009). The NimStim set of facial expressions: judgments from untrained research participants. Psychiatry Research, 168, 242-249.

Weisgerber, A., Vermeulen, N., Peretz, I., Samson, S., Philippot, P., Maurage, P., ... Constant, E. (2015). Facial, vocal and musical emotion recognition is altered in paranoid schizophrenic patients. Psychiatry Research, 229, 188-193.

Wilhelm, O., Hildebrandt, A., Manske, K., Schacht, A., \& Sommer, W. (2014). Test battery for measuring the perception and recognition of facial expressions of emotion. Frontiers in Psychology, 5, 404. 
